\title{
Reintervention in Endodontics
}

Author(s)/Editor(s): Mario Luiz Zuolo / Daniel Kherlakian / José Eduardo de Mello Jr / Maria Cristina Coelho de Carvalho / Maria Inês Ranazzi Cabral Fagundes

Publisher: Quintessence Editora - Sao Paulo, Brazil

Language: English

ISBN: 978-85-7889-042-1

Edition: $1 / \mathrm{e}$

Publish Year: 2014

Pages: 332, illustrated

Price: $\$ 180.00$

DOI: https://doi.org/10.25241/stomaeduj.2016.3(3-4).bookreview.3

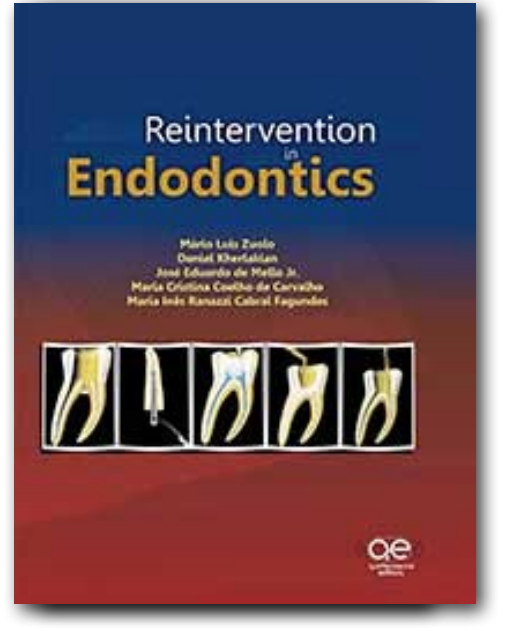

Ateam of Brazilian specialists wrote this book to help practitioners improvethe quality of the endodontic retreatment. Because nowadays compromised retreatments are no longer an option this book together with the latest materials, tools, and technologic resources increases the predictability and quality of endodontic reinterventions. This book is divided into thirteen chapters. The introduction speaks about the definition and purpose of endodontic retreatments. The indications and contraindications for non-surgical endodontic retreatment are presented in the next chapter. The tools needed in this type of treatment are discussed in chapter three. The following four chapters cover endodontic access, removal of intra radicular posts, techniques for removing gutta-percha and solid materials. Techniques for repreparation of the root canal system are presented in a separate chapter. Steps, materials and techniques for refilling the canal system are very well described. Prevention and treatments protocols for mishaps and special cases are tackled in two other chapters. Restoration after endodontic retreatment and predictability rates of endodontics versus implantodontics are discussed in the final chapters. The authors manage to write a concise text and to providea complete description of the treatment protocols for a wide variety of reintervention cases. The book also covers all the relevant topics in a simple manner and is accompanied by more than 1,350 illustrations. This book is necessary to dental practitioners and endodontists to help them find the best solution for treatment in reintervention cases.

The Books Review is drafted in the reviewer's sole wording and illustrates his opinions.

Florin Eugen

Constantinescu

DDS, PhDStudent

ROPOSTURO-Holistic Dental

Medicine Institute

Bucharest, Romania

e-mail:

dr.florin.constantinescu@gmail.com 